\title{
Near Infrared Spectrophotometric Method for the Determination of Hydration Numbers
}

\author{
Richard A. Durst* and John K. Taylor
}

(July 29, 1964)

\begin{abstract}
The $980 \mathrm{~m} \mu$ absorption band of water is employed in a new method for the determination of hydration numbers. The decrease in the concentration of "free" water is calculated from the change in absorbance resulting on the addition of an electrolyte. Data are given for chromic chloride and nitrate salts in water-methanol solutions, and an extrapolation is made to give the hydration number in pure water. The results obtained by this technique agree with the values reported using other methods.
\end{abstract}

\section{Introduction}

Although aqueous electrolyte solutions have been studied in detail for many years, one of the most basic properties of such solutions, the concept of ionic hydration, is still poorly defined. Normally, the effect of the coordinated water molecules is ignored when reactions in aqueous solutions are considered. However, in many cases, this can lead to serious errors in the interpretation of results. The numerous experimental techniques which have been employed to determine hydration numbers give some indication of the widespread influence of ion-solvent interactions. These methods include such phenomena as: transference of ions, freezing and boiling points, vapor pressure, density, compressibility, and refractivity [1], ${ }^{1}$ to mention a few. In addition, knowledge of hydration numbers is important for development of a theory of concentrated electrolyte solutions and for the theory of reaction in solution.

\section{Hydration Number Concept}

Part of the difficulty in defining absolute hydration numbers is the indefinite nature of the term "hydration number" itself. It is generally accepted that ions possess two hydration spheres. The primary hydration sphere is made up of those water molecules which are firmly associated with the ion by an electrostatic ion-dipole attraction and have lost their own separate translational degrees of freedom. The secondary hydration sphere contains water molecules which undergo interaction partially with the primary hydration sphere of the ion and

*Present Address: Dept. of Chemistry, Pomona College, Claremont, Cali Figures in brackets indicate the literature references at the end of this paper. partially with the surrounding water structure. This sphere extends an indefinite distance from the ion until it ultimately blends into the bulk water structure. Normally, the secondary hydration is very weak and values found experimentally vary widely depending on the phenomenon observed.

To date, the techniques employed for determining. hydration numbers have resulted in values which can be divided into two groups: low values which seem to approximate the primary hydration numbers and considerably larger values corresponding to the primary hydration plus all or part of the secondary hydration sphere. Since it is the primary hydration number which is important as far as the properties of the ionic entity are concerned, the method used must not be so sensitive as to include contributions from the secondary hydration.

The hydration techniques may be further subdivided into those which measure some property of the solvated ions and those which measure some property of the solvent itself. In the former grouping may be included such phenomena as polarography [2, 3], UV-visible spectrophotometry [4], mobility [5], and activity [6] measurements, while the second grouping includes isotope dilution [7], compressibilities [8, 9], and nuclear magnetic resonance [10].

\section{Technique}

The method proposed here is one of the latter type based on the near infrared (NIR) absorption of water. Water exhibits a series of absorption bands in the NIR from about 1 to $3 \mu$. Using the band peak between $980 \mathrm{~m} \mu$ and $1020 \mathrm{~m} \mu$, which is the third overtone of the $\mathrm{OH}$ stretching vibration [11], changes in the free water concentration in solution will be measured when an electrolyte is introduced. The 
change in the free water concentration can then be related to the water tied up in the primary hydration sphere of the ions.

Methanol solutions containing varying amounts of water were prepared for the purpose of calibration. A second identical set of solutions was then prepared containing a known concentration of electrolyte and comparison was made of the water absorption in the 980 to $1020 \mathrm{~m} \mu$ region. The apparent decrease in the water concentration was attributed to a shift of this absorption band for the water molecules in the hydration sphere of the ions. This shift is caused by the strong interaction of the water dipole with the electric field surrounding the ion. In this region, the water molecules are highly compressed by the strong electrostatic field and, consequently, it is assumed that the $\mathrm{OH}$ stretching vibrational energy will be significantly affected. Since the water molecules in the secondary hydration sphere interact very weakly with the hydrated ions, their absorption band closely coincides with the free water band and no separation occurs. Thus the secondary hydration does not cause any apparent water concentration changes.

Since hydrogen bonding in this solvent system causes association to occur, there is the possibility that the absorption changes produced when the electrolyte is added is due to a disassociation effect rather than to hydration. However, it has been demonstrated that the absorption of the water band at $0.96 \mu$ increases with disassociation [12]. Since the effect observed upon the addition of the chromium salts is just the opposite, i.e., a decrease in the absorption, the electrolyte affect on the solvent association can be ruled out as a major factor in this study. To verify this further, it is proposed that a salt such as $\mathrm{NH}_{4} \mathrm{ClO}_{4}$ be used in a subsequent study. This electrolyte should exhibit a hydration number of almost zero and thus not effect the absorption of the water band to any significant extent. In this way, the contribution due to association changes can be evaluated.

It is evident that the number of moles of water in the primary hydration sphere can be calculated from the decrease in the absorption band. This is accomplished by obtaining from the calibration curve the mole percent free water remaining after the salt is added and converting to moles of water. This number is then subtracted from the moles of water originally present to give the total number of moles of hydrating water. This value divided by the moles of electrolyte present must, therefore, be the hydration number of the electrolyte. By choosing an electrolyte composed of a small cation of high charge and a large anion of low charge, the hydration effect may be confined primarily to the cation. If this were not done, the total hydration number of both the cation and anion would be obtained and the individual ionic hydration numbers could not be distinguished.

In order to evaluate the method, chromic ion was selected as the cation since several independent methods $[7,13]$ have shown this ion to have an inert primary hydration sphere of 6 water molecules.
For the preliminary investigation, $\mathrm{CrCl}_{3} \cdot 6 \mathrm{H}_{2} \mathrm{O}$ was used. The chloride ion hydration is small, being on the order of 0 to 1 and should not be significant in the dilute water in methanol solutions. Subsequently, the nitrate salt was employed since its hydration is weaker than that of chloride. The best anion in this respect would be perchlorate. However, since this salt is only available with an indefinite number of waters of hydration, it could not be used without causing serious error in the hydration number measured.

Methanol (A.C.S., 99.8\% purity) was selected as the solvent since it is completely miscible with water and has a low dielectric constant (31.5 to 32.6 at $\left.25^{\circ} \mathrm{C}[14]\right)$. Because of its low dielectric constant, it will not compete strongly with water $(\epsilon=78.5$ at 25 $\left.{ }^{\circ} \mathrm{C}[14]\right)$ for the primary solvation sphere of the ion and will be displaced at relatively low water concentrations.

\section{Apparatus and Procedure}

The instrument used in this investigation was a Perkin-Elmer Corp. Spectracord Model 4000A. The methanol-water solutions were placed in $10 \mathrm{~cm}$ cells, and the spectra scanned from 1200 to $800 \mathrm{~m} \mu$.

In the preliminary work with reagent grade $\mathrm{CrCl}_{3} \cdot 6 \mathrm{H}_{2} \mathrm{O}$, the Spectracord was operated as a single-beam spectrophotometer. Using a slit width of $0.40 \mathrm{~mm}$, a series of spectra were recorded for methanol-water solutions from 0.0 to 72.1 mole percent water. Several such spectra superimposed are represented in figure 1 . The absorbance of the band peak was plotted versus the water concentration for the purpose of calibration, and this curve is given in figure 2 . The transmittance scale was arbitrarily set by instrumental adjustment so that the methanol band at $1020 \mathrm{~m} \mu$ had a value of approximately 0.80 . This was done to bring the methanolwater bands into the more sensitive transmittance region. This technique explains the resulting transmittance greater than 1.00 on either side of the absorption band. Using this scale, the arbitrary transmittance values $(\bar{T})$ obtained were converted into the "absorbance" units $(\bar{A})$ plotted in the empirical working curve given in figure 2 .

The solutions containing chromic chloride were then run in the same way giving spectra of the type shown in figure 3 . The only differences in the spectra of these solutions and the calibration solutions are the apparent decrease in the water concentration and the rapid cut off at short wavelengths due to chromic ion absorption. By means of the calibration curve, the apparent water concentration of these solutions is measured, and the difference found is attributed to the removal of water from the bulk solution by hydration.

Subsequent work was carried out using chromic nitrate salts and operating the Spectracord in the double beam mode. In these cases, methanol was used as the reference medium while the sample solutions were similar to those used in the case of chromic chloride. By means of the double beam 


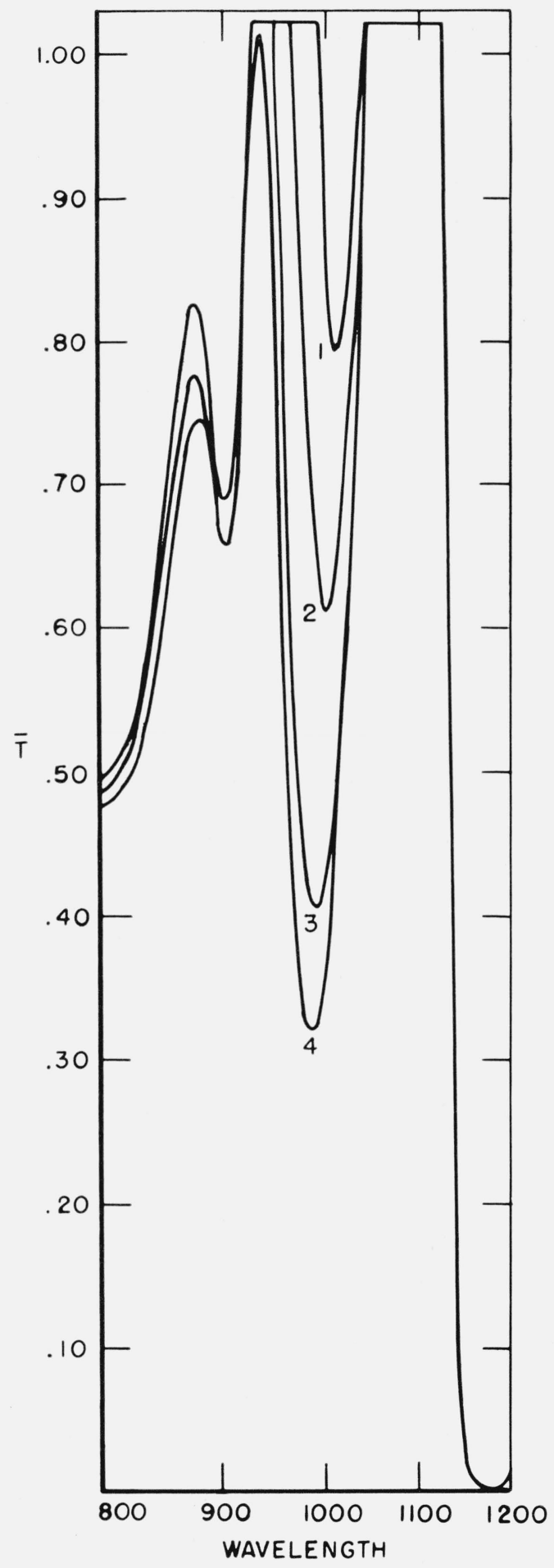

Figure 1. $\mathrm{H}_{2} \mathrm{O}$ absorption band in $\mathrm{H}_{2} \mathrm{O}-\mathrm{CH}_{3} \mathrm{OH}$ solutions. (1) $\mathrm{CH}_{3} \mathrm{OH}$

(2) 23.4 mole percent $\mathrm{H}_{2} \mathrm{O}$

(3) 43.6 mole percent $\mathrm{H}_{2} \mathrm{O}$

(4) 53.8 mole percent $\mathrm{H}_{2} \mathrm{O}$ operation, the major portion of the background in the spectra was eliminated as can be seen in figure 4 (curve No. 1). Again, to increase the sensitivity, the transmittance at $1 \mu$ was arbitrarily set at approximately 0.90 for pure methanol. The calibration spectra and those for the sample solutions were all recorded on the same chart in order to facilitate comparison (fig. 4). In figure 5, the calibration curve is given for the water "absorbance" versus mole percent water in methanol when operating in the double beam mode. By comparison with figure 2 , it is seen that the sensitivity of the method is increased by the use of the double beam technique. In all cases, the solutions were rerun several times over the period of a week to ensure equilibration of the aquo complexes. As expected, no changes were noted as a function of time indicating a rapid attainment of equilibrium.

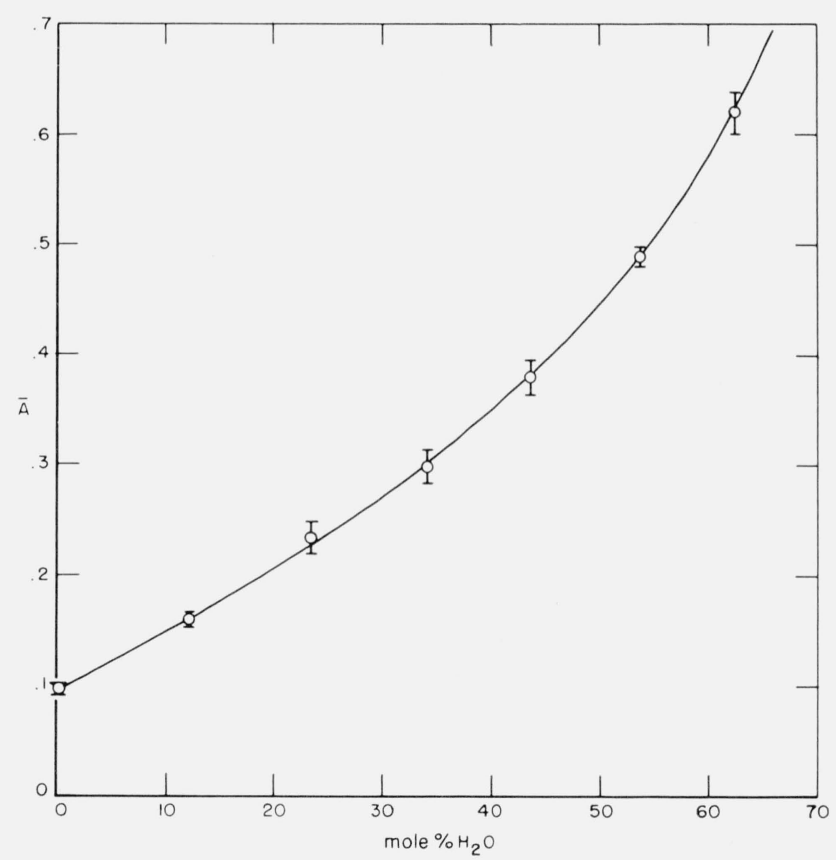

Figure 2. Absorbance of $\mathrm{H}_{2} \mathrm{O}$ between 980 and 1020 m $\mu$ versus mole percent water (single beam operation).

\section{Results}

\subsection{Chromic Chloride}

The approximately $0.5 \mathrm{M} \mathrm{CrCl}_{3} \cdot 6 \mathrm{H}_{2} \mathrm{O}$ solutions were prepared containing 0.05 mole of $\mathrm{Cr}^{+++}$in the methanol-water solvents. The water concentration, including the contribution due to the waters of hydration of the salt, was varied from 12.1 to 69.5 mole percent. Data were gathered at seven water concentrations, and the results with the standard deviation of the means are given in table 1 . The number of observations reported for each concentration refers to independent measurements on aliquots of a stock solution. 

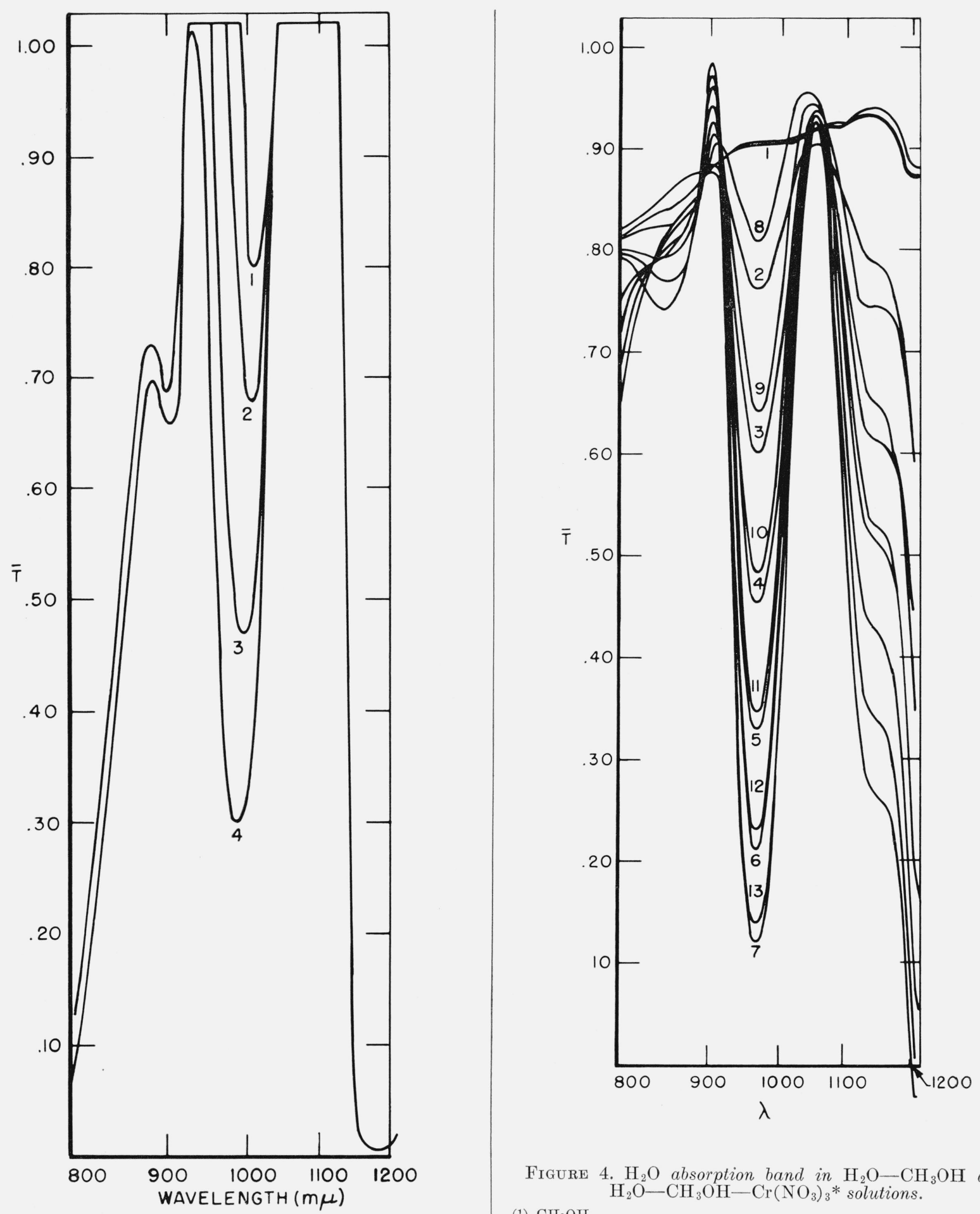

Figure 4. $\mathrm{H}_{2} \mathrm{O}$ absorption band in $\mathrm{H}_{2} \mathrm{O}-\mathrm{CH}_{3} \mathrm{OH}$ and $\mathrm{H}_{2} \mathrm{O}-\mathrm{CH}_{3} \mathrm{OH}-\mathrm{Cr}\left(\mathrm{NO}_{3}\right)_{3}$ * solutions.

(1) $\mathrm{CH}_{3} \mathrm{OH}$

percent $\mathrm{H}_{2} \mathrm{O}$

(3) 20 mole percent $\mathrm{H}_{2} \mathrm{O}$

(4) 30 mole percent $\mathrm{H}_{2} \mathrm{O}$

(5) 40 mole percent $\mathrm{H}_{2} \mathrm{O}$

(6) 50 mole percent $\mathrm{H}_{2} \mathrm{O}$

(7) 60 mole percent $\mathrm{H}_{2} \mathrm{O}$

(8) 10 mole percent $\mathrm{H}_{2} \mathrm{O}+\mathrm{Cr}\left(\mathrm{NO}_{3}\right)_{3}$

(9) 20 mole percent $\mathrm{H}_{2} \mathrm{O}+\mathrm{Cr}\left(\mathrm{NO}_{3}\right)_{3}$

(1i) 30 mole percent $\left.\mathrm{H}_{2} \mathrm{O}+\mathrm{Cr}_{2} \mathrm{NO}_{3}\right)_{3}$

(11) 40 mole percent $\mathrm{H}_{2} \mathrm{O}+\mathrm{Cr}\left(\mathrm{NO}_{3}\right)_{3}$

(12) 50 mole percent $\mathrm{H}_{2} \mathrm{O}+\mathrm{Cr}\left(\mathrm{NO}_{3}\right)_{3}$

(2) 123.4 mole percent $\mathrm{H}_{2} \mathrm{O}$

(4) 62.5 mole percent $\mathrm{H}_{2} \mathrm{O}$

*0.05 mole $\mathrm{CrCl}_{3}$

*0.02 mole $\mathrm{Cr}\left(\mathrm{NO}_{3}\right)_{3}$ 


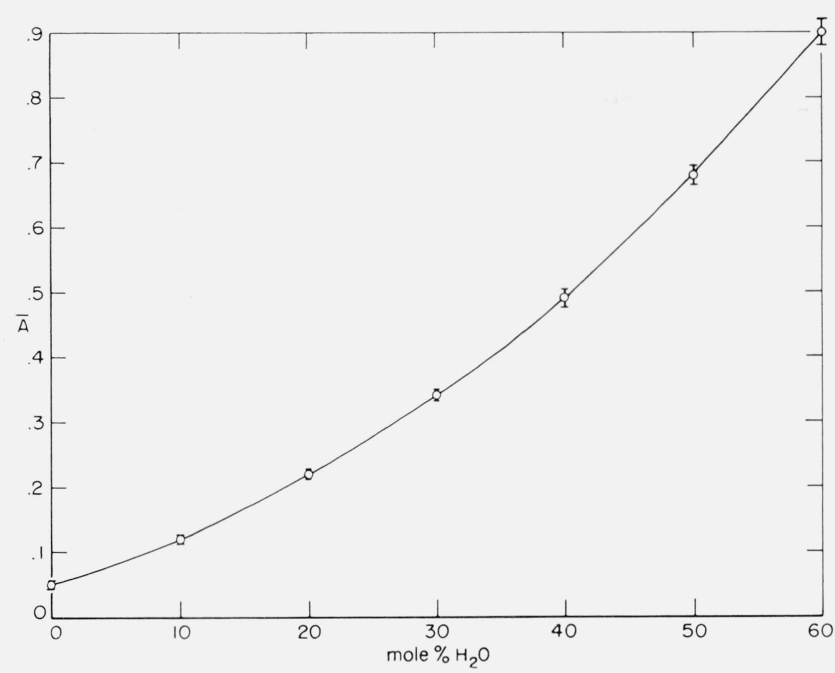

Figure 5. Absorbance of $\mathrm{H}_{2} \mathrm{O}$ at 980 mu versus mole percent $\mathrm{H}_{2} \mathrm{O}$ in $\mathrm{CH}_{3} \mathrm{OH}$ (double beam operation).

These seven points lie along a straight line which when extrapolated to 100 mole percent water results in a hydration number for $\mathrm{CrCl}_{3}$ of $5.7_{2} \pm 0.1_{6}$.

This value is the sum of the hydration numbers for chromic and chloride ions. However, since data are taken at lower water concentrations and the degree of chloride ion hydration is small (hyd. No. $=0-1$ ), the effect of chloride hydration is very likely negligible. Thus, the hydration number found is primarily due to chromic ion hydration.

TABLE 1. $\mathrm{CrCl}_{3} \cdot 6 \mathrm{H}_{2} \mathrm{O}$ results

\begin{tabular}{c|c|c|c|c}
\hline \hline [Cr++] & $\begin{array}{c}\text { Mole } \\
\text { percent } \\
\mathrm{H}_{2} \mathrm{O}\end{array}$ & $\begin{array}{c}\text { Hydration } \\
\text { No. }\end{array}$ & $\begin{array}{c}\text { Number of } \\
\text { observations }\end{array}$ & $\begin{array}{c}\text { Standard } \\
\text { deviation } \\
\text { of the mean }\end{array}$ \\
\cline { 1 - 2 } Moles/liter & & & & \\
0.59 & 12.1 & 4.7 & 7 & 0.2 \\
.56 & 23.4 & 4.8 & 5 & .09 \\
.52 & 34.0 & 4.9 & 8 & $.0_{6}$ \\
.50 & 43.4 & 5.0 & 7 & .1 \\
.50 & 51.8 & 5.1 & 9 & $.11_{1}$ \\
.50 & 60.4 & 5.3 & 11 & .18 \\
.50 & 69.5 & 5.4 & 11 & $.11_{4}$ \\
\hline
\end{tabular}

A disadvantage of using chromic chloride is the complication resulting from the formation of chlorochromium complexes. At the concentrations used, only the monochlorochromium complex formation is significant. From the formation constant, $\mathrm{K}_{\mathrm{CrCl}^{++}}=$ 0.1 , it is calculated that 0.06 mole of $\mathrm{CrCl}^{++}$is present and, as a first approximation, should lower the accepted hydration number of 6 for noncomplexed chromic ion by about 2.0 percent. This would result in a hydration number of $5.8_{8}$; a value within the standard deviation of the hydration number experimentally found.

\subsection{Chromic Nitrate}

To avoid the difficulties arising from complex formation, subsequent work was carried out with chromic nitrate salts. Solutions were prepared con- taining 0.02 mole of reagent grade $\mathrm{Cr}\left(\mathrm{NO}_{3}\right)_{3} \cdot 9 \mathrm{H}_{2} \mathrm{O}$ in methanol with the water concentration varied from 10 to 50 mole percent. The results of these hydration number determinations are given in table 2 .

When the line through these points is extrapolated to 100 mole percent water, the resulting hydration number is $6.6_{5} \pm 0.3_{4}$. This value is higher than expected: possibly due to hydration of the nitrate ion. As in the case of chloride, the nitrate hydration should be small (less than 1), but the larger value for the hydration number of the nitrate salt indicates that its total hydration is greater although weaker than that of the chloride ion and, as will be seen, only becomes significant when the $\mathrm{H}_{2} \mathrm{O}$ concentration is relatively high.

TABLE 2. $\mathrm{Cr}\left(\mathrm{NO}_{3}\right)_{3} \cdot 9 \mathrm{H}_{2} \mathrm{O}$ results

\begin{tabular}{c|c|c|c|c}
\hline \hline$\left[\mathrm{Cr}^{+++}\right]$ & $\begin{array}{c}\text { Mole } \\
\text { percent } \\
\mathrm{H}_{2} \mathrm{O}\end{array}$ & $\begin{array}{c}\text { Hydration } \\
\text { No. }\end{array}$ & $\begin{array}{c}\text { Number of } \\
\text { observations }\end{array}$ & $\begin{array}{c}\text { Standard } \\
\text { deviation of } \\
\text { the mean }\end{array}$ \\
\cline { 1 - 1 } Moles/liter & & & & \\
0.24 & 10 & 3.5 & 7 & \\
.22 & 20 & 3.5 & 12 & 0.08 \\
.21 & 30 & 4.1 & 13 & .13 \\
.20 & 40 & 4.4 & 13 & .1 \\
.20 & 50 & 4.8 & 13 & .12 \\
\hline
\end{tabular}

Another reagent grade nitrate salt, $\mathrm{Cr}\left(\mathrm{NO}_{3}\right)_{3} \cdot 7 \frac{1}{2}$ $\mathrm{H}_{2} \mathrm{O}$, was used for the final determination. The solutions were made up to contain 0.04 mole of this salt and water concentrations from 18.7 to 60.0 mole percent. These results are given in table 3.

A line through these points extrapolates to $7.1_{6} \pm 0.1_{7}$ at 100 mole percent water. Again, this value is larger than expected but, as before, is probably due to nitrate hydration and elimination of complexation by the anion which causes a lowering of the hydration number.

TABLE 3. $\mathrm{Cr}\left(\mathrm{NO}_{3}\right)_{3} \cdot 7 \frac{1}{2} \mathrm{H}_{2} \mathrm{O}$ results

\begin{tabular}{c|c|c|c|c}
\hline \hline [Cr & $\begin{array}{c}\text { Mole } \\
\text { percent } \\
\mathrm{H}_{2} \mathrm{O}\end{array}$ & $\begin{array}{c}\text { Hydration } \\
\text { No. }\end{array}$ & $\begin{array}{c}\text { Number of } \\
\text { observations }\end{array}$ & $\begin{array}{c}\text { Standard } \\
\text { deviation of } \\
\text { the mean }\end{array}$ \\
\hline Moles/liter & & & & \\
0.44 & 18.7 & 2.5 & 8 & 0.07 \\
.42 & 29.0 & 3.0 & 14 & .1 \\
.40 & 39.3 & 3.6 & 14 & .07 \\
.40 & 49.7 & 4.2 & 14 & .08 \\
.40 & 60.0 & 4.9 & 17 & .09 \\
\hline
\end{tabular}

\section{Discussion and Conclusion}

The data for the three salts investigated are plotted in figure 6. Although the chromic salts exhibit large differences in the hydration numbers at low water concentrations, the differences decrease at higher concentrations. That is, at 50 mole percent $\mathrm{H}_{2} \mathrm{O}$, all three salts have hydration numbers between 4 and 5 and, at 60 and 70 mole percent $\mathrm{H}_{2} \mathrm{O}$, the agreement is even closer. Then, however, at even higher water concentrations, the values again appear to diverge, but since these values are in the extrapolation region of the graph, the actual divergence could be much less. 


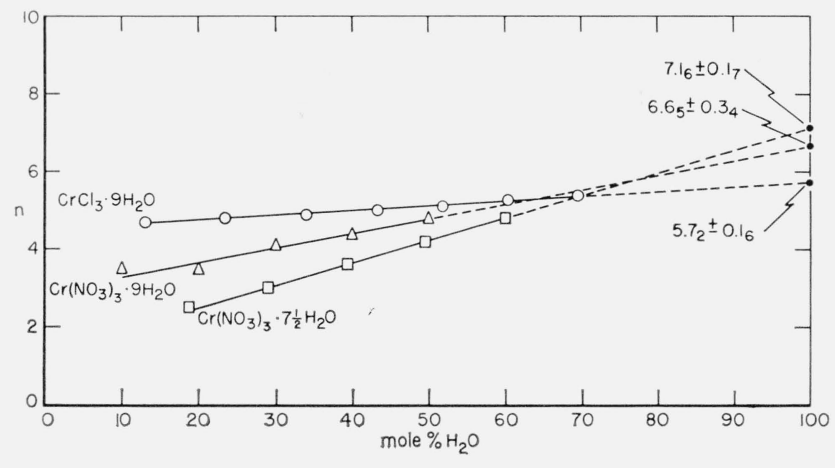

Figure 6. Hydration numbers for chromic salts versus mole percent $\mathrm{H}_{2} \mathrm{O}$.

The large divergence in hydration number values at low water concentrations is to be expected, since in this range, there is a strong competition by the ions present for the small amount of water. Thus it would appear that the chloride ion hydrates more readily than nitrate. This results in the higher apparent hydration number for the chloride salt with respect to the nitrate salts. At higher water concentrations where competition for water is not so strong, the nitrate begins to hydrate and rapidly approaches the chloride salt hydration number. At least three explanations for the increase in the chromic nitrate hydration number over the chromic chloride are possible: (1) the total effective hydration number of nitrate is greater than chloride, (2) the complexation of chromic ion with chloride reduces the number of possible hydration sites around both the chromic and chloride ions, and (3) the extrapolation to 100 mole percent water is invalid and the actual hydration number for the nitrate salts levels off above a concentration of about 60 mole percent of $\mathrm{H}_{2} \mathrm{O}$. In the case of (1), it is necessary that the chloride ion hydration be stronger than the nitrate, so that the nitrate hydration is not detected until the water concentration is relatively high. Although it is definitely known that case (2) does occur, the extent to which it lowers the hydration number cannot be predicted with any degree of certainty. Finally, in order to test the effect of case (3), it would be necessary to run chromic nitrate solutions at water concentrations greater than 60 mole percent. However, at these concentrations, the precision of the measurements is extremely low since the method used measures the change in the absorption of free water. Thus it can be seen that any one or combination of these possibilities could be the cause of the divergence in the extrapolated high water concentration region.

The values determined for the chromic ion in the method evaluation experiments further support the results found by other hydration number techniques. The precision of the hydration number values obtained by this technique compares very favorably with those from the existing methods and can be improved with minor modifications. Limitations on the method were set by such factors as the reproducibility of the instrument and errors in the composition of the hydrated salts.

The validity of the extrapolation of the hydration numbers in mixed solvent systems, such as $\mathrm{H}_{2} \mathrm{O}-\mathrm{CH}_{3} \mathrm{OH}$, to pure water can be checked by employing solvents with dielectric constants greater and lesser than that for methanol. This should show any solvent effects on the hydrated ions. Of course, to demonstrate the validity of the extrapolation, the extrapolated value should be independent of the solvent used. A better choice than methanol might have been acetonitrile $\left(\epsilon=36.7\right.$ at $25{ }^{\circ} \mathrm{C}$ [14]) since this solvent does not have an absorption band in the $1 \mu$ region and there is no complicating $\mathrm{OH}$ type bonding. However, since methanol-water working curves were used, the effects caused by overlapping bands are thereby taken into consideration. Anion and salt concentration effects must also be clarified before the method can be used for any survey of the hydration numbers of diverse ions.

Since it is the concentration of water and not the particular ion which is measured, this method should be applicable to the determination of the hydration numbers of any ions which form relatively inert aquo complexes, i.e., hydrated ions, and do not have interfering absorption bands in the NIR region. Labile or weak aquo complexes probably would not exhibit large enough separation between the complexing and free water bands and only result in broadening the existing free water absorption bands in the NIR. In spite of the limitations noted, the purpose of this preliminary study has been achieved; that of the development of a new, independent method for the determination of primary hydration numbers.

\section{References}

[1] J. O’ M. Bockris, Quart. Revs. (London) 3, 173 (1949).

[2] K. P. Mishchenko and I. S. Pominov, Zhur. Fiz. Khim. 31, 2026 (1957).

[3] I. S. Pominov, Ibid. 31, 2184 (1957).

[4] J. Bjerrum and C. K. Jorgensen, Acta Chem. Scand. $\boldsymbol{y}$, 951 (1953).

[5] H. Ulich, Trans. Faraday Soc. 23, 392 (1927).

[6] P. O. Kosakewich and N. A. Ismailow, Z. Physik. Chem. (Leipzig) 150, A, 308 (1930).

[7] J. P. Hunt and H. Taube, J. Chem. Phys. 19, 602 (1951).

[8] A. L. J. Moesveld and H. J. Hardon, Z. physik. Chem. (Leipzig) 155, A, 238 (1931).

[9] A. Passinski, Acta Physicochim. U.R.S.S. 8, 385 (1938).

[10] J. A. Jackson, J. F. Lemons, and H. Taube, J. Chem. Phys. 32, $553(1960)$.

[11] W. Kaye, Spectrochim. Acta 6, 257 (1954).

[12] W. Luck, Berichte Bunsengesellschaft physik. Chem. 6\%, 186 (1963)

[13] P. B. D. de la Mare and W. Klyne, Progress in Stereochemistry, V. III (Butterworths, Washington, D.C., 1962).

[14] R. A. Robinson and R. H. Stokes, Electrolyte Solutions (Butterworths, London, 1959). 